Fermilab-TM-2353-AD

October, 2001

\title{
Proposal for Continuously-Variable Neutrino Beam Energy for the NuMI Facility
}

\author{
Mikhail Kostin, Sacha Kopp \\ Department of Physics, University of Texas, Austin, TX 78712 \\ Mark Messier \\ Harvard University, Cambridge, MA 02138 \\ Debbie Harris, Jim Hylen, Adam Para \\ Fermi National Accelerator Laboratory, Batavia, IL 60510
}

\begin{abstract}
The NuMI Facility was intended to be flexibly changed between 3 energies of beams, LE, ME, and HE. However, the changeover requires extensive downtime to move and realign horns and the target. We propose to implement a flexible arrangement where the target can be remotely moved in the beamline direction to change the beam energy and the horns remain fixed. In addition to having the attractive feature of keeping the horn optics fixed, the motion of the target can be performed more quickly and hence on a more frequent basis. We discuss potential increases in statistics in the high energy region, systematic cross-checks available, and the improved beam monitoring capabilities with such variable energy beams.
\end{abstract}

\section{Introduction}

The NuMI Facility was designed with an extra-long target hall so that the horns could be moved to achieve 3 different beam energies, the low energy (LE) beam with $E \sim 3 \mathrm{GeV}$, the medium energy (ME) beam with $E \sim 6 \mathrm{GeV}$, and the high energy (HE) beam with $E \sim 15 \mathrm{GeV}$. In the original design [1], Horn 1 remains fixed at a location along the beamline, while horn 2 moves further downstream along the beamline to move to the higher beam energies (see Figure 1). Furthermore, in order to switch to higher energy beams, the target is moved further upstream. In the LE beam, the upstream face of the target is at $z=-35 \mathrm{~cm}$, in ME beam it is $-1.3 \mathrm{~m}$, and in the $\mathrm{HE}$ beam it is at $-3.96 \mathrm{~m}$. The ME and HE beams use a different target from the LE target.

We propose to add a new flexibility to the beamline, that of being able to vary the beam energy by leaving the 2 horns fixed at their LE position and remotely moving the LE target back in $z$. As will be discussed in greater detail in Section 3 and shown in Figure 1, simply retracting the LE target out of horn one by $1-3 \mathrm{~m}$ can achieve a poor-man's ME or HE beam, or any energy in between. Because the target for the LE beam has to be cantilivered inside horn 1, such a sliding insertion/retraction mechanism is already being developed, but 


\section{GNUMI V-14}

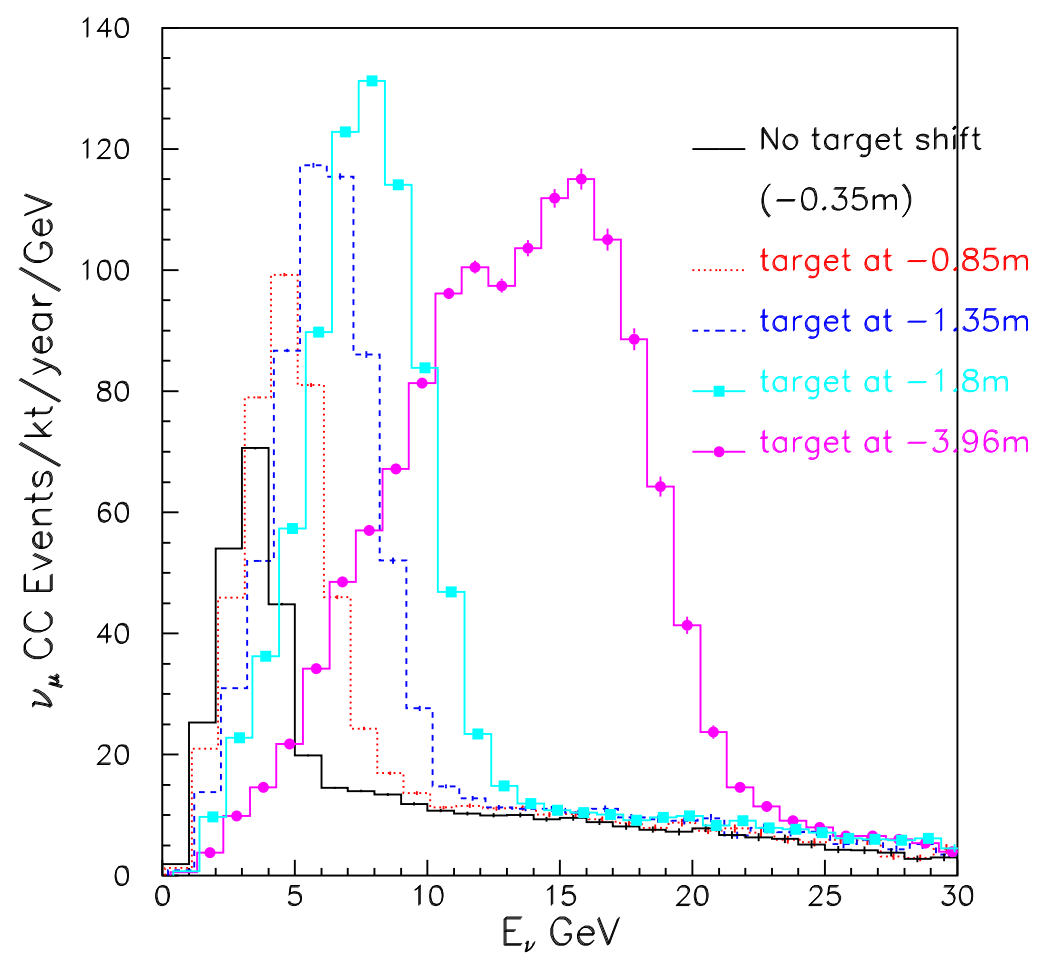

Figure 1: Neutrino event rate in the far detector generated with the two horns in the LE beam position and with the LE target located at $z$ of $-0.35,-0.85,-1.35,-1.8$, and $-3.96 \mathrm{~m}$.

is not presently motorized. As we will show, automating this feature and possibly extending its range to $-4 \mathrm{~m}$ may have advantages for flexible beam running. In this section we outline some of the advantages of such a poor-man's, or "Semi-ME" and "Semi-HE" beam. In the following sections we provide more detailed simulations of how it compares to the nominal $\mathrm{LE} / \mathrm{ME} / \mathrm{HE}$ beams. While the target can be scanned continuously to any position with such an arrangement, we have simulated relocating the target to the equivalent positions for $\mathrm{ME}$ and HE beams for ease of comparison. The principle reasons for pursuing a variable-energy beam via relocating the target are:

- The semi-ME and semi-HE beams would increase the statistics in the 6-20 GeV neutrino energy region. It may be decided, for reasons of physics or experimental checks, that increasing the statistics in this region would be important (such as, for example, to discriminate between conventional oscillation models and neutrino decay models, where the region above $6 \mathrm{GeV}$ is helpful). As is clear from Figure 1, a 2 kton-year run of the semi-HE beam accumulates as many events as 8 kton-years of LE running around $10 \mathrm{GeV}$. The collaboration could decide on a hybrid dataset of combined LE, semi-ME, and semi-HE runs. 

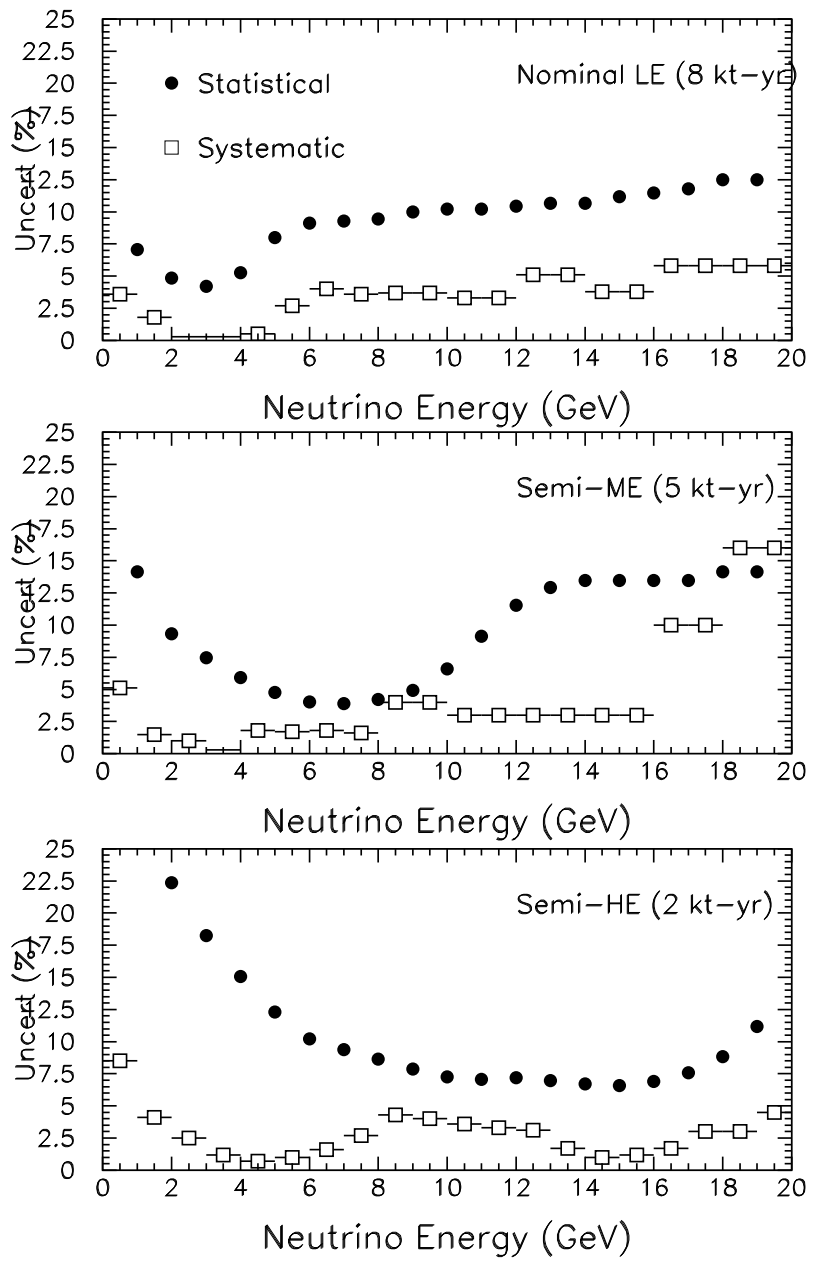

Figure 2: Statistical and systematic uncertainties from hadron production models in the beams obtained with the LE target located at $z$ of -0.35 (nominal LE), -1.35 (semi-ME), and $-3.96 \mathrm{~m}$ (semi-HE) beams. In this calculation, it was assumed there would be $8 \mathrm{kt}-\mathrm{yr}$ of LE, 5 kt-yr of semi-ME, and 2 kt-yr of semi-HE running.

- It is reasonably quick to change between the LE beam and these other semi-ME/HE beams. As discussed in Section 6, the changeover requires only remote manipulation of the target. The horns do not require relocation and realignment, which saves 2-3 months downtime for the switch. It may be imagined that MINOS' initial data running in the LE beam may have physics surprises that would make rapid reaction to move to $\mathrm{ME} / \mathrm{HE}$ beam desirable, or even more likely it may be imagined that hints of systematic effects (muon monitor asymmetries, near detector spectral distortion) would make very desirable a cross-check in one of the semi-ME/HE, where such systematics would have predictable effects.

The downtime and cost for relocating horns has been cited as one of the reasons the collaboration would not readily opt for a mix of LE/ME/HE runs. 

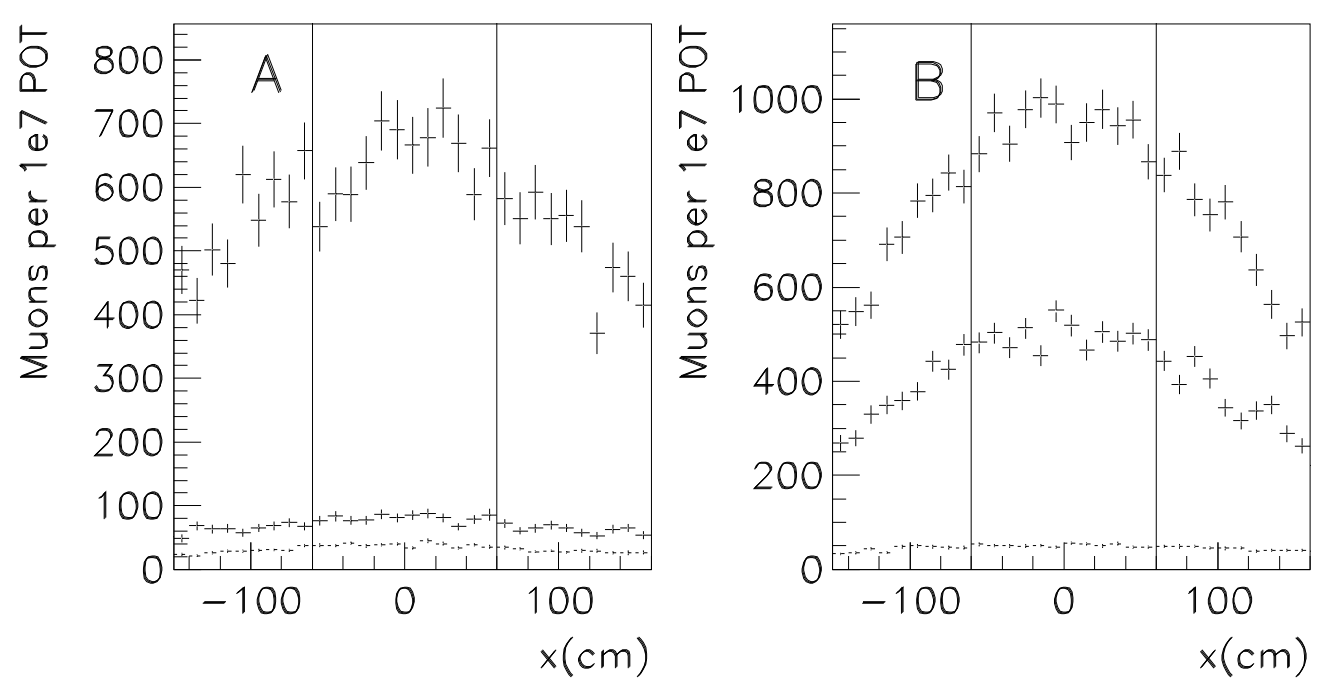

Figure 3: (left) Muon distribution in the 3 alcoves for the nominal LE beam. (right) Muon distribution in the 3 alcoves for the Semi-ME beam.

- Overall systematic errors from the beam could also be lowered with a combined LE + semi-ME/HE run as opposed to flat-out LE running. This lowering of systematics comes in several categories. First, by comparing the same energy bin from the LE and the semi-ME or semi-HE data set, one has a chance to compare the same bin from two data sets with very different systematics. Many of these systematics are the lowest in the focusing peaks of whatever beam one is running. So, running the higher energy beam may give us the best measurement of the neutrino disappearance probability from the point of view of systematics as well.

An example of such systematic errors is shown in Figure 2, where the statistical uncertainties and the systematic uncertainties from hadron production in the target [4] are compared for a $8 \mathrm{kt}-\mathrm{yr}$ LE run, a $5 \mathrm{kt}$-yr run with the target at $-1.35 \mathrm{~m}$ (semi-ME), and a 2 kt-yr run with the target at $-4 \mathrm{~m}$ (semi-HE run). This figure suggests that the most optimal uncertainties would be obtained using the $0-4 \mathrm{GeV}$ data from the LE beam, the 4-11 GeV data from the semi-ME beam and the 11-20 GeV data from the semi-HE beam.

The systematics are also lower in comparing data sets because the horns remain fixed at all times, so that their optics are not changed during the run. Only the target moves, so only the relative alignment between the target and horns needs to be checked when switching beams. This is not the case if MINOS decided to run with a mix of the conventional LE/ME/HE beams.

We discuss systematic errors in the conventional LE/ME/HE beams as well as the semi-ME/HE beams further in Section 4. 
- Significant benefit to the commissioning of the beam would be added if the variable target position were implemented. One of the significant problems with using the hadron and muon monitoring systems in pointing the neutrino beam to Soudan or otherwise diagnosing problems during the commissioning of the beam is that the LE beam is so broad. The ME and HE beams, in contrast, are more collimated, so that even within the narrow acceptance of the muon monitors one can see a peak in the muon distribution corresponding to the center of the neutrino beam direction. This peaking is all the more important because only the central $\pm 60 \mathrm{~cm}$ around the beamline can be used in the monitors for pointing the neutrino beam due to the complex geometry of the hadron absorber which is upstream of the muon system (see Section 5). As shown in Figure 3, the muon distribution in the first two alcoves peaks within $60 \mathrm{~cm}$ in the semi-ME beam, while it is essentially flat in the broad LE beam.

- Finally, beam monitoring could perhaps better be accomplished by occaisionally reverting to the "Semi-ME" or "Semi-HE" Beam during data taking. This periodic, short run could be used to check that nothing in the beam has changed and is more sensitive than using the LE beam running monitoring data. This point is discussed further in Section 5 .

The remainder of this note provides some back-up studies for the discussion outlined in this section. Section 2 provides more detail on the geometry of the target hall, the targets used in the conventional LE/ME/HE beams, and target+horn positions for these beams. Section 3 compares the event rates in the semi-ME/HE beams with the conventional $\mathrm{ME} / \mathrm{HE}$ beams which were optimized for event rate by switching to a different target and by telescoping horn 2. Section 4 compares systematic uncertainties in the semi-ME/HE beams to those in the conventional LE/ME/HE beams. Section 5 further studies monitoring capabilities in the semi-ME/HE beams, and Section 6 discusses the mechanics now in the baseline to retract the LE target by as much as $1 \mathrm{~m}$ (to the ME position) and the adaptations that would be needed to achieve a $4 \mathrm{~m}$ shift. Section 7 concludes and lists several avenues for investigation and discussion.

\section{Beam Geometry}

Running at different neutrino beam energies by moving the target in and out of the horn can obviously be done at any target position and thus one can continuously select the peak energy of the neutrino distribution. For purposes of comparison with the nominal LE/ME/HE beams, we have simulated in this note the nominal beams, and also the beam obtained by moving the LE target backwards by $1.0 \mathrm{~m}$ (the "semi-ME" beam), and by moving the target backwards by $3.96 \mathrm{~m}$ (the "semi-HE" beam) to more directly compare with the nominal ME and HE beams.

Figure 4 shows the layout of the target hall for each of the nominal LE, ME, and HE beams. The locations of the various elements are specified in Table 1. In this coordinate 


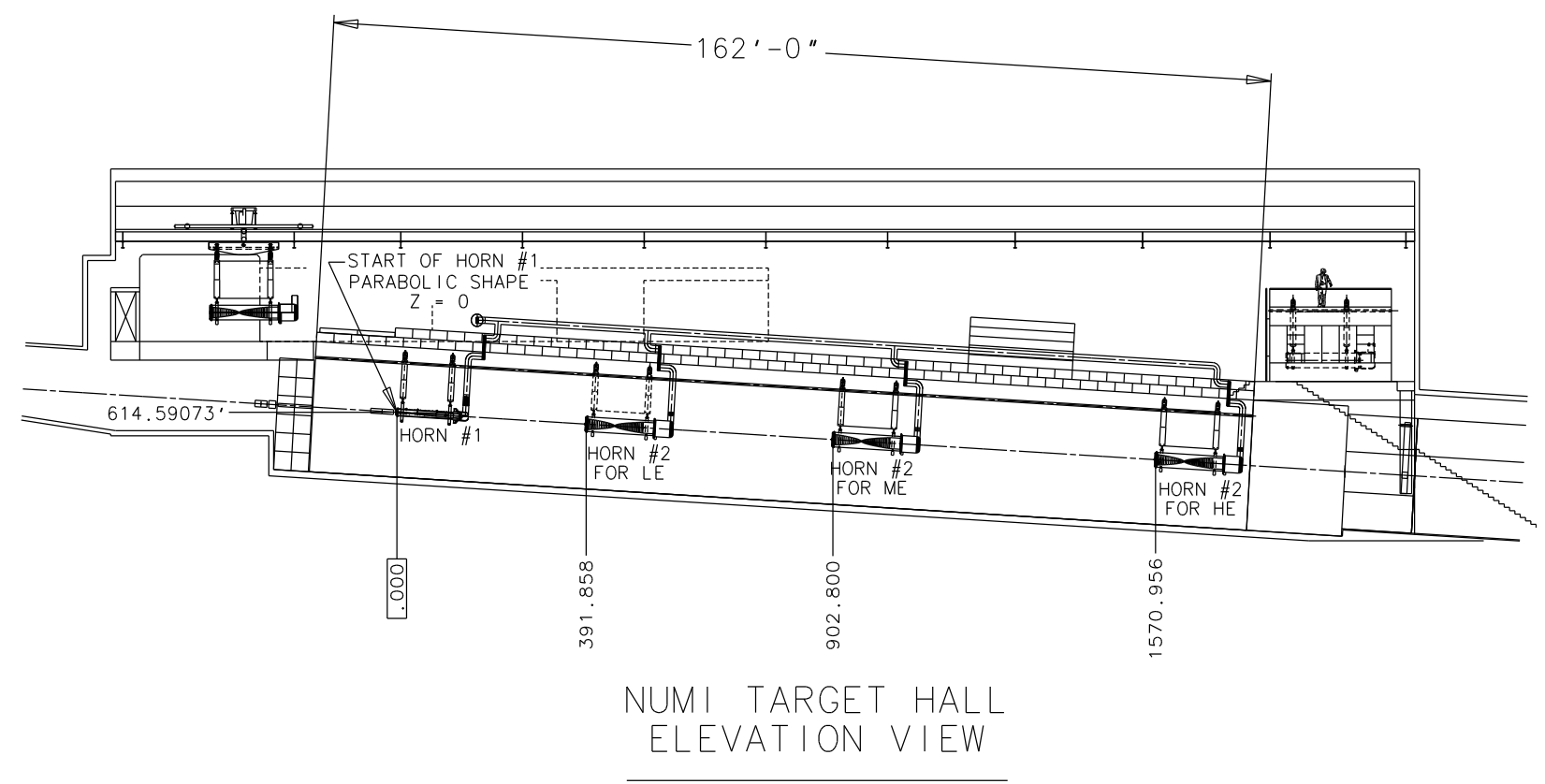

Figure 4: The NuMI target hall showing Horn 1 located at $z=0 \mathrm{~m}$, with Horn 2 located at 9,23 , or $40 \mathrm{~m}$ for the LE, ME, or HE beams. All the $z$ locations in the figure are given in inches.

system, $z=0 \mathrm{~m}$ is the location of the upstream end of horn 1 . The nominal LE beam uses a different target than the ME and HE beams, the latter two sharing the same target design. In the variable energy beam obtained by scanning the target in $z$, we would not swap out the target, but merely retract it backwards to a new $z$ location.

In the baseline beam design, inserting the target inside the horn for LE beam requires that it will be mounted on a travel mechanism that allows it to slide into horn 1 once the target module is lowered into the target hall. The target can retract naturally as much as $1 \mathrm{~m}$ behind its LE position without hitting the baffle. If a larger target travel were desired, moving the baffle would be required (and would be required for the nominal HE beam). This takes a little more time than just moving the target (see Section 6.

Table 2 gives the parameters of the targets simulated in this note. These targets are rectangular solids meant to approximate in some way the true 'fin' geometry of the targets. For example, the actual LE target is actually a series of $4720 \times 6.4 \times 20 \mathrm{~mm}^{3}$ fins with $0.3 \mathrm{~mm}$ gaps in between them. Thus, we scaled the expected $1.68 \mathrm{~g} / \mathrm{cm}^{3}$ density of this target by $20 / 20.3$ to obtain a final density of $1.66 \mathrm{~g} / \mathrm{cm}^{3}$ density. The ME/HE target has larger gaps, so its average density is lower in the simulation, $1.41 \mathrm{~g} / \mathrm{cm}^{3}$. It is made of narrower and taller fins, although the proton beam hits the fins $3.8 \mathrm{~cm}$ off-center in the vertical direction (the lower part of the fins being used to support the fins in a clamp).

All of the simulations performed in this note were done with Gnumi Version 14.0 [3], unless otherwise noted. 


\begin{tabular}{cccc} 
Beam & Target type & Target $Z_{0}(\mathrm{~m})$ & Horn 2 position $(\mathrm{m})$ \\
\hline \hline Nominal LE & LE & -0.35 & 10 \\
Nominal ME & $\mathrm{ME}$ & -1.3 & 23 \\
"Semi-ME" & $\mathrm{LE}$ & -1.35 & 10 \\
Nominal HE & $\mathrm{ME}$ & -3.96 & 40 \\
"Semi-HE" & $\mathrm{LE}$ & -3.96 & 10 \\
\hline
\end{tabular}

Table 1: Parameters of the beams simulated in this note. Target type is describe in Table 2, Target $Z_{0}$ is the position of the upstream edge of the target $(z=0 \mathrm{~m}$ is at horn 1$)$.

\section{Event Rates in the Far Detector}

The semi-ME and semi-HE beams do not achieve the full event rates possible in the nominal ME/HE beams. Figure 5 shows the event rates in the far detector per kt-yr in the "semi" beams and in the nominal ME, HE beams. As can be seen, the semi-ME and semi-HE beam produce $\sim 70-80 \%$ of the usual event rate of the ME and HE beams. It is instructive to investigate where is the origin of $20-30 \%$ loss in event rate.

First we note that the semi-ME and semi-HE beams do not utilize the optimized ME/HE target, but instead use the LE target because this requires the least downtime for switching configurations. The effect of the non-optimized target is studied in Figure 6. In this figure, the semi-beams are generated with the LE target as in Figure 5, and also with the ME/HE target. The upstream end of the target is the same for the semi-HE simulations $(z=$ $-3.96 \mathrm{~m})$, and for the semi-ME simulations there is a $5 \mathrm{~cm}$ difference $(z=-1.35 \mathrm{~m}$ for the LE target and $z=-1.3 \mathrm{~m}$ for the ME target) owing to the different target lengths.

In the case of the ME configuration, the semi-beam loses about $20 \%$ of the event rate to the true ME beam (120 events in the peak relative to 150). As seen from Figure 6, only a negligible fraction of this is due to the use of the LE target in the semi-ME beam. The dominant effect is apparently the fact that horn 2 was not 'telescoped' forward as would be done for the true ME beam (as shown explicitly in Figure 7).

In the case of the HE beam, the target and lack of telescoping of horn 2 both appear relevant. The semi-HE beam has approximately 30\% fewer events in the peak (105 vs 150) relative to the true HE beam. As shown in Figure 6, the use of the LE target in the semi-HE beam is responsible for $10 \%$ or more of the $30 \%$ drop. As shown in Figure 7 , the inability to telescope horn 2 hurts the HE event rate by an additional $15 \%$.

\begin{tabular}{c|c|c} 
Geometry Parameter & LE Target & ME/HE Target \\
\hline \hline Length $(\mathrm{m})$ & 0.95 & 1.20 \\
Height $(\mathrm{mm})$ & 20 & 80 \\
Width $(\mathrm{mm})$ & 6.4 & 3.2 \\
Density $(\mathrm{g} / \mathrm{cc})$ & 1.66 & 1.41 \\
\hline
\end{tabular}

Table 2: Parameters of the LE and ME targets in the simulations. 


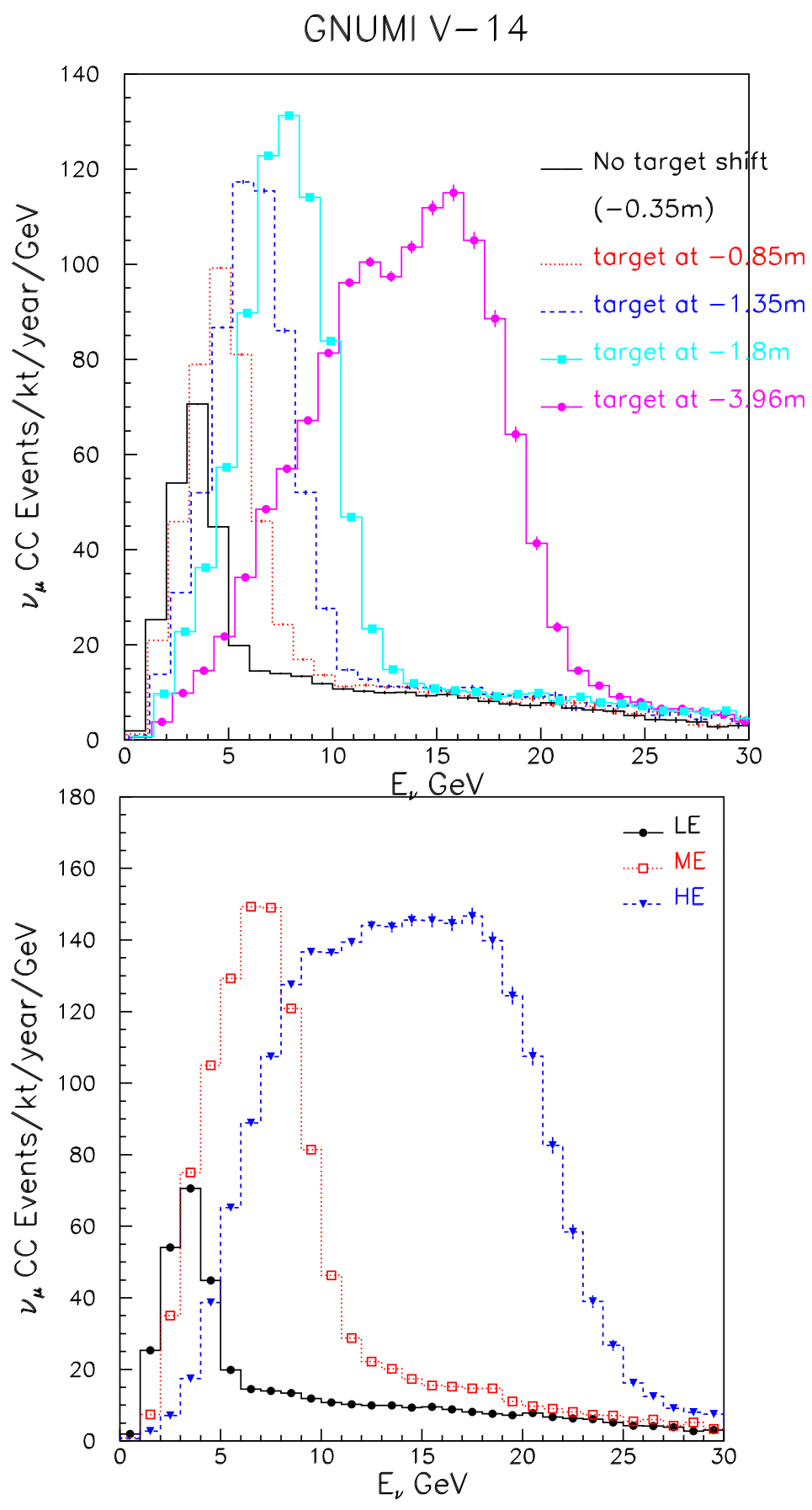

Figure 5: (top) Neutrino event rate in the far detector generated with the two horns in the LE beam position and with the LE target located at $z$ of $-0.35,-0.85,-1.35,-1.8$, and $-3.96 \mathrm{~m}$. (bottom) The far detector event rate in the conventional LE/ME/HE beams. 

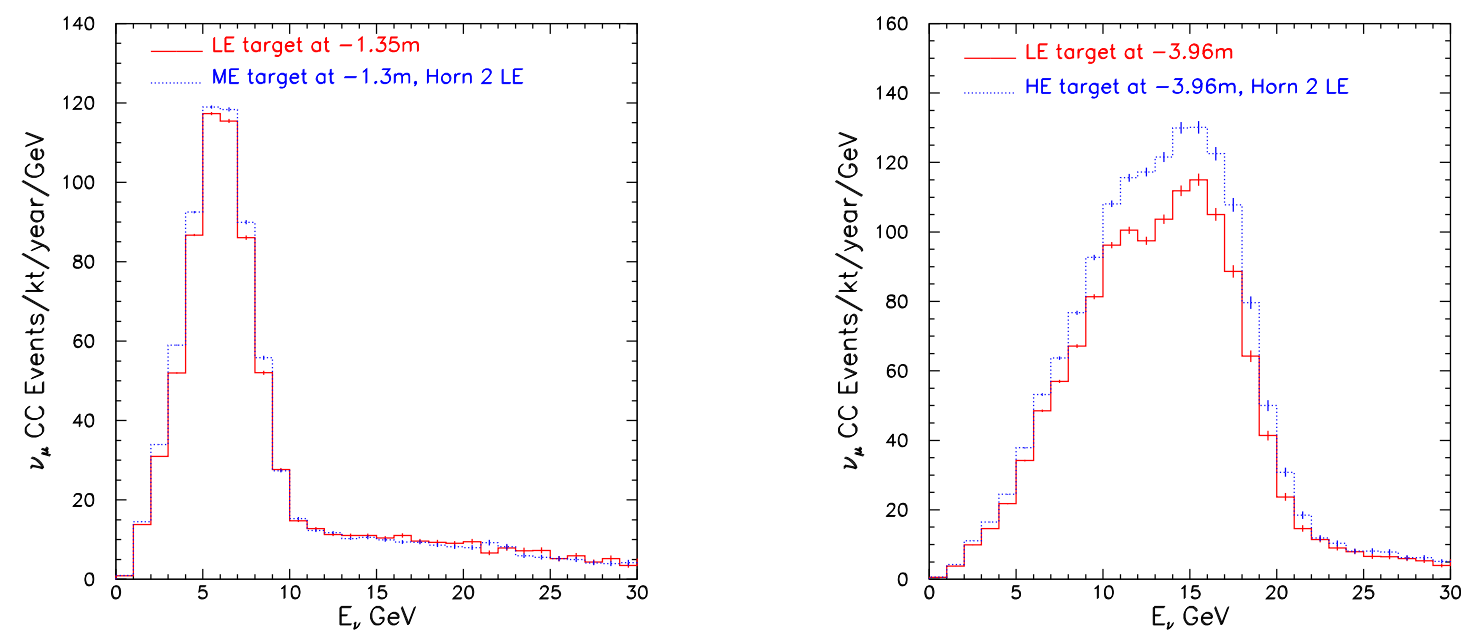

Figure 6: Comparison of the semi-ME beam (left) and semi-HE beam (right) generated with the LE target (red curves) and with the ME/HE target (blue curves). Evidently, the target geometry has a small impact on the event rate in the far detector for the medium-energy beam, but a noticeable impact on the high-energy beam.
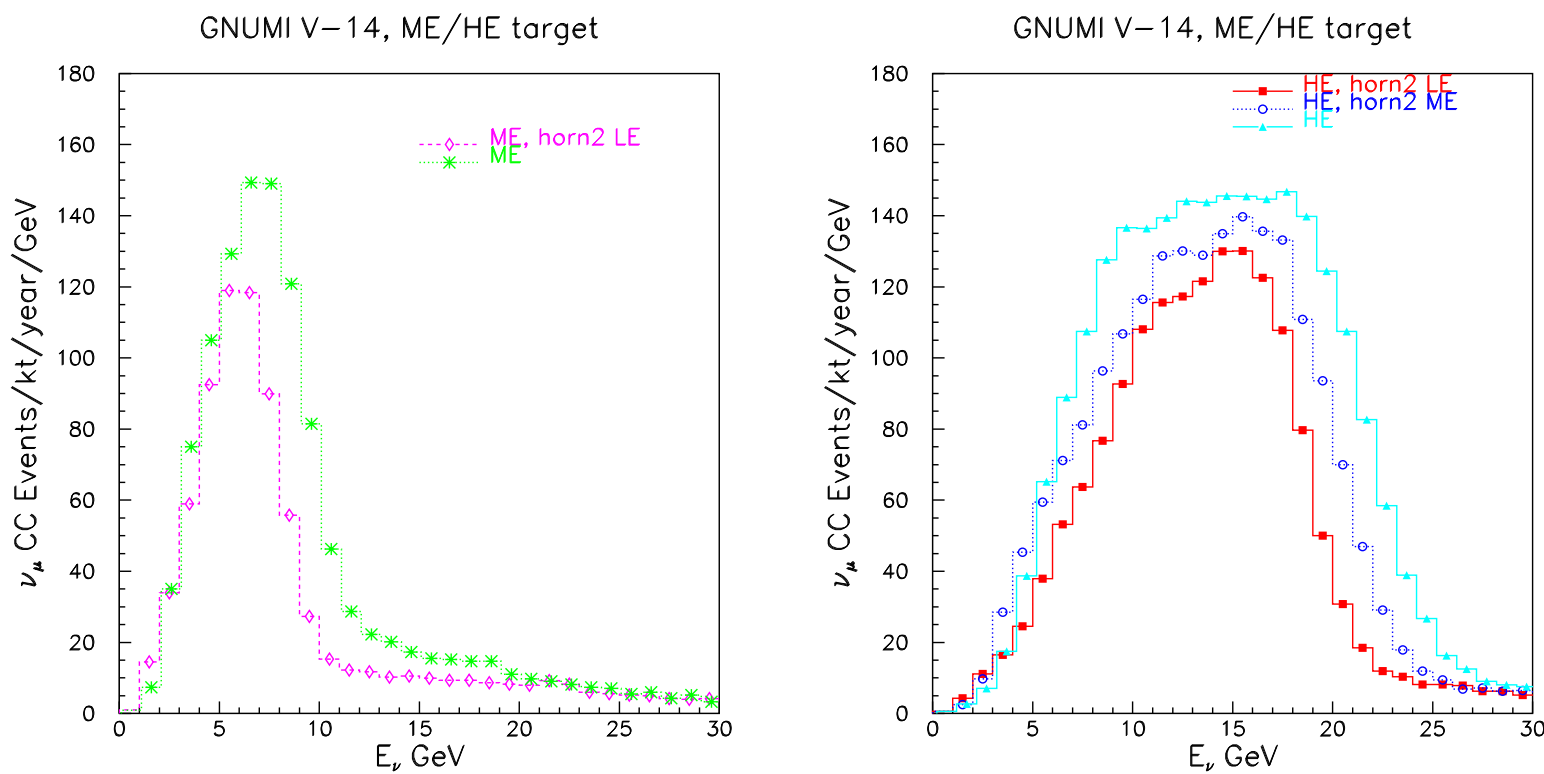

Figure 7: (left) The far detector event rate generated with the ME target at the ME position $(z=-1.3 \mathrm{~m})$ with horn 2 at the ME or the LE positions. (right) The far detector event rate generated with the ME target at the HE postion $(-3.96 \mathrm{~m})$ and horn 2 at either the LE, ME, or HE positions. Evidently, the position of Horn 2 has a strong impact on the event rate in the far detector for both the medium- and high-energy beams. 

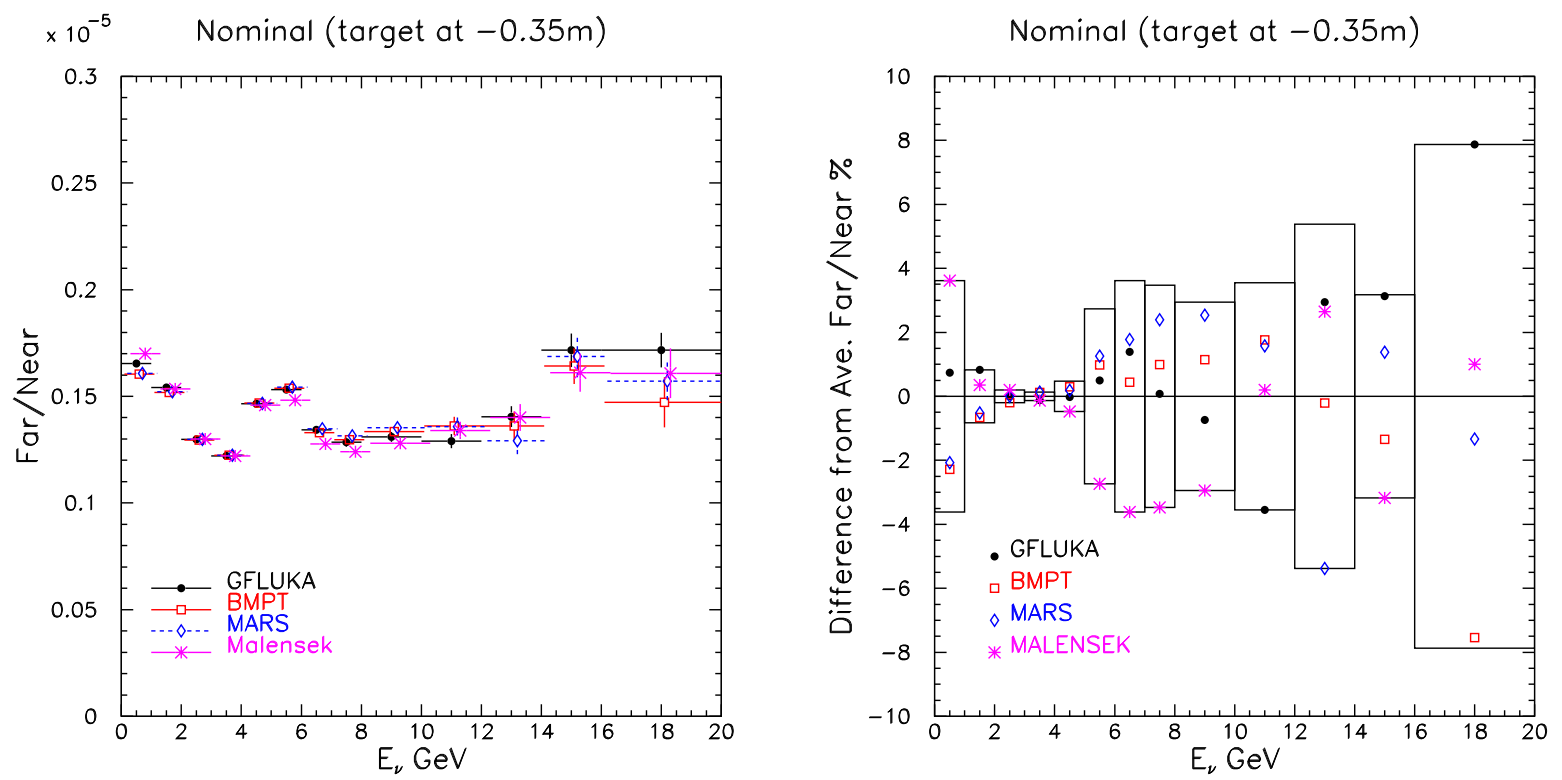

Figure 8: (left) The variation in far-over-near ratio expected due to different hadron production models in the LE beam. The lowest systematic spread from this source always occurs in the peak. (right) The $\mathrm{F} / \mathrm{N}$ of several models relative to that of Geant/Fluka.

\section{Systematic Uncertainties}

An additional reason to implement the rapid-cycling semi-ME and semi-HE beams is to trade off statistics in the low energy bins for lower systematics in the higher energy bins. In the nominal LE beam, the largest systematic uncertainties in the spectrum always result in the so-called 'high energy tail', where one falls off the focusing peaks of the horns. The high energy tail should provide a control or normalization region where oscillations are not expected. In some exotic neutrino models, such as extra dimensions or neutrino decay, this 6-20 GeV region is also useful for demonstrating that the neutrino disappearance is not due to one of these more exotic models.

With the importance of this region in mind, it might be desirable to cycle back and forth between the LE beam and the semi-ME or semi-HE beam, obtaining lower systematics in these semi-beam runs for the $6-20 \mathrm{GeV}$ range. Systematic uncertainties should always be lowest in the focusing peaks of a beam, be it a LE, ME, or HE beam. Thus, by going to the semi-ME or semi-HE beam, we could obtain a short run which would give us our best measurement of survival probability in the higher neutrino energy bins.

We have investigate one particular systematic uncertainty, the spread due to the hadron production error [4]. The MARS13 [5], Geant/Fluka [6], Malensek [8], and BMPT [7] models are considered. Figure 8 shows the spread in Far/Near for the nominal LE beam. We subsequently investigate how such uncertainties manifest themselves in the higher-energy beams. 
Figure 9 and 10 show the systematic error from hadron production in the true ME and the semi-ME beam. Despite the poorer focusing the the semi-ME beam, the error spread does not appear to be substantially larger in the semi-ME beam. Likewise, the model spread in the semi-HE beam is not substantially larger than the semi-HE beam. And, in both cases, the systematic spread is smaller than that from the true LE beam in the range $6<E_{\nu}<15 \mathrm{GeV}$ (see Figure 8). There is interesting structure in the error bands from hadron production when comparing, for example, the nominal ME and the semi-ME beam, or the nominal HE to the semi-HE beam. This is presumably due to the different location of the horn 2 focusing element, but must be studied further.

Another example of a very different sort of systematic effect is shown in Figure 13. In this figure is simulated the effect in Far/Near ratio for the nominal LE beam, the semi-ME beam, and the semi-HE beam when horn 1 is misaligned by $3 \mathrm{~mm}$. The vertical axis is the ratio of Far/Near in this misaligned case to the Far/Near expected for the aligned beam. The deviation of these plots from 1.0 represents a systematic error to the experiment if such an effect were not discovered. As has been noted by others, this and many other alignment systematics grow much larger for ME/HE beams than in the LE beam. So, if any odd effects, such as a change in spectrum in the near detector or an asymmetry in the muon chambers, were noted during LE running, having the capability to switch rapidly to a semi-ME/HE beam would be very useful to try to cross-check and understand the origin of the noted effect. Many misalignments are generally acceptable to run with, as long as they are known and understood. 

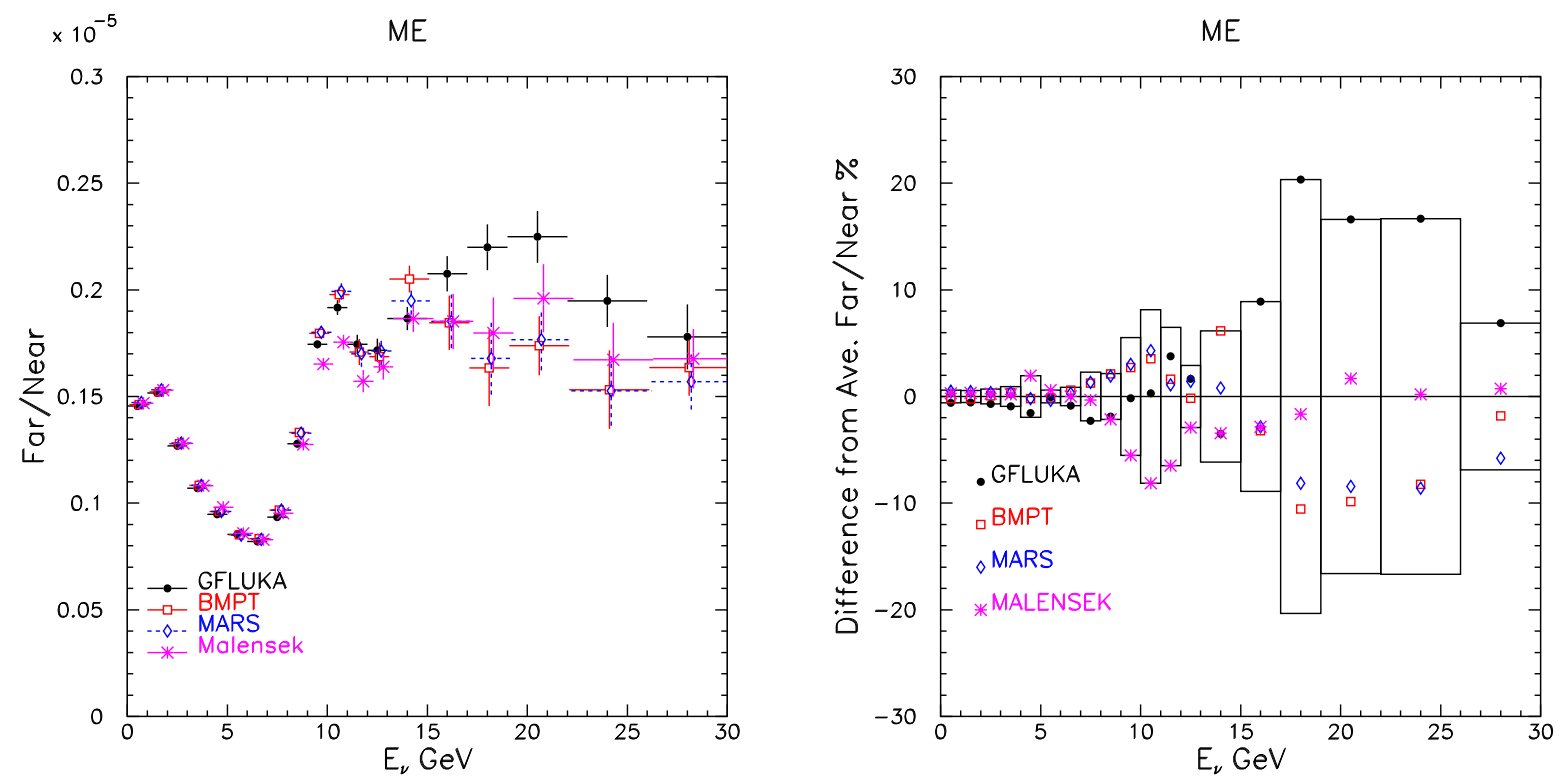

Figure 9: (left) The variation in far-over-near ratio expected due to different hadron production models in the ME beam. The lowest systematic spread from this source always occurs in the peak. (right) The $\mathrm{F} / \mathrm{N}$ of several models relative to that of Geant/Fluka.
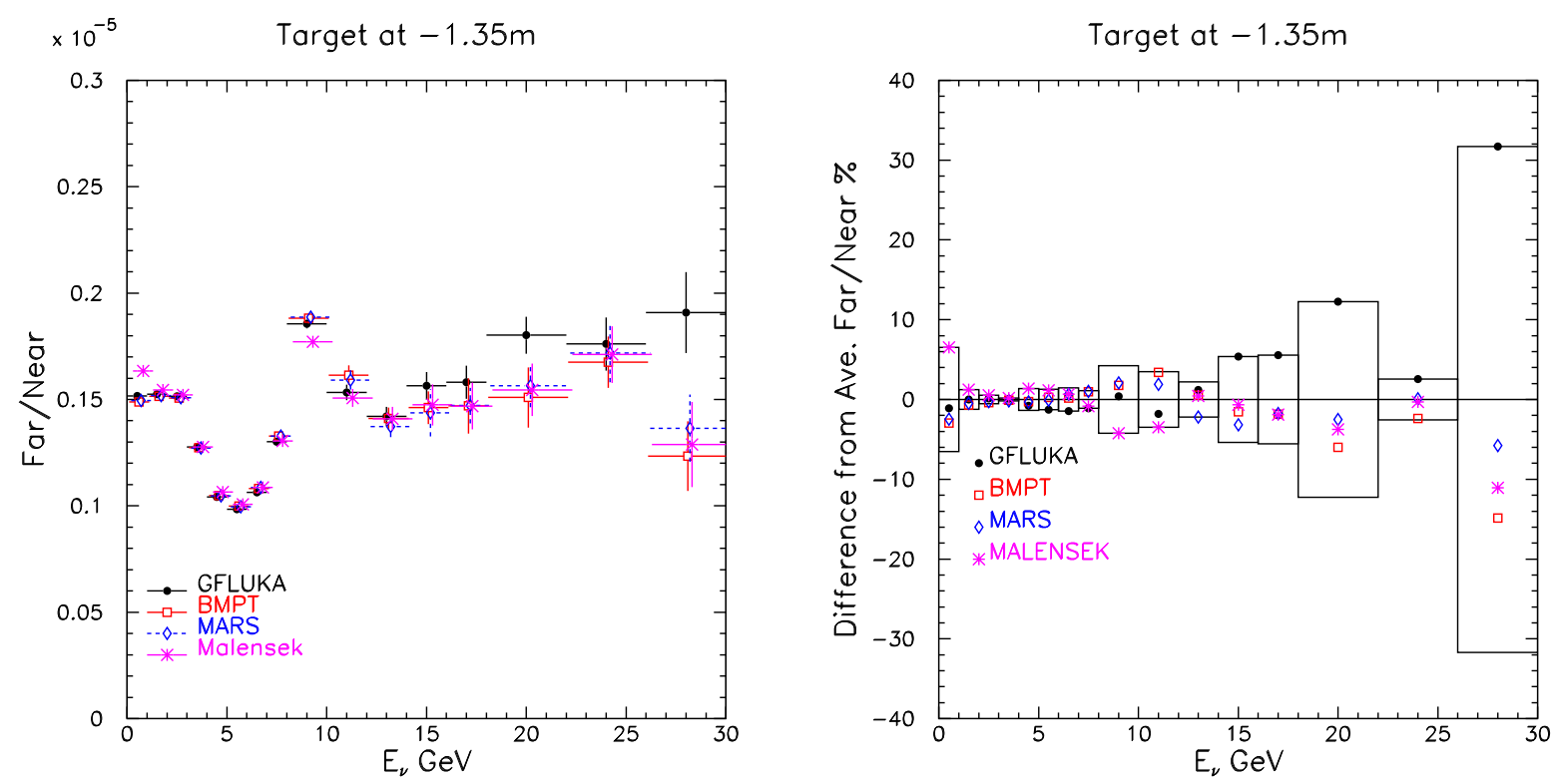

Figure 10: (left) The variation in far-over-near ratio expected due to different hadron production models in the semi-ME beam. The lowest systematic spread from this source always occurs in the peak. (right) The $\mathrm{F} / \mathrm{N}$ of several models relative to that of Geant/Fluka. 

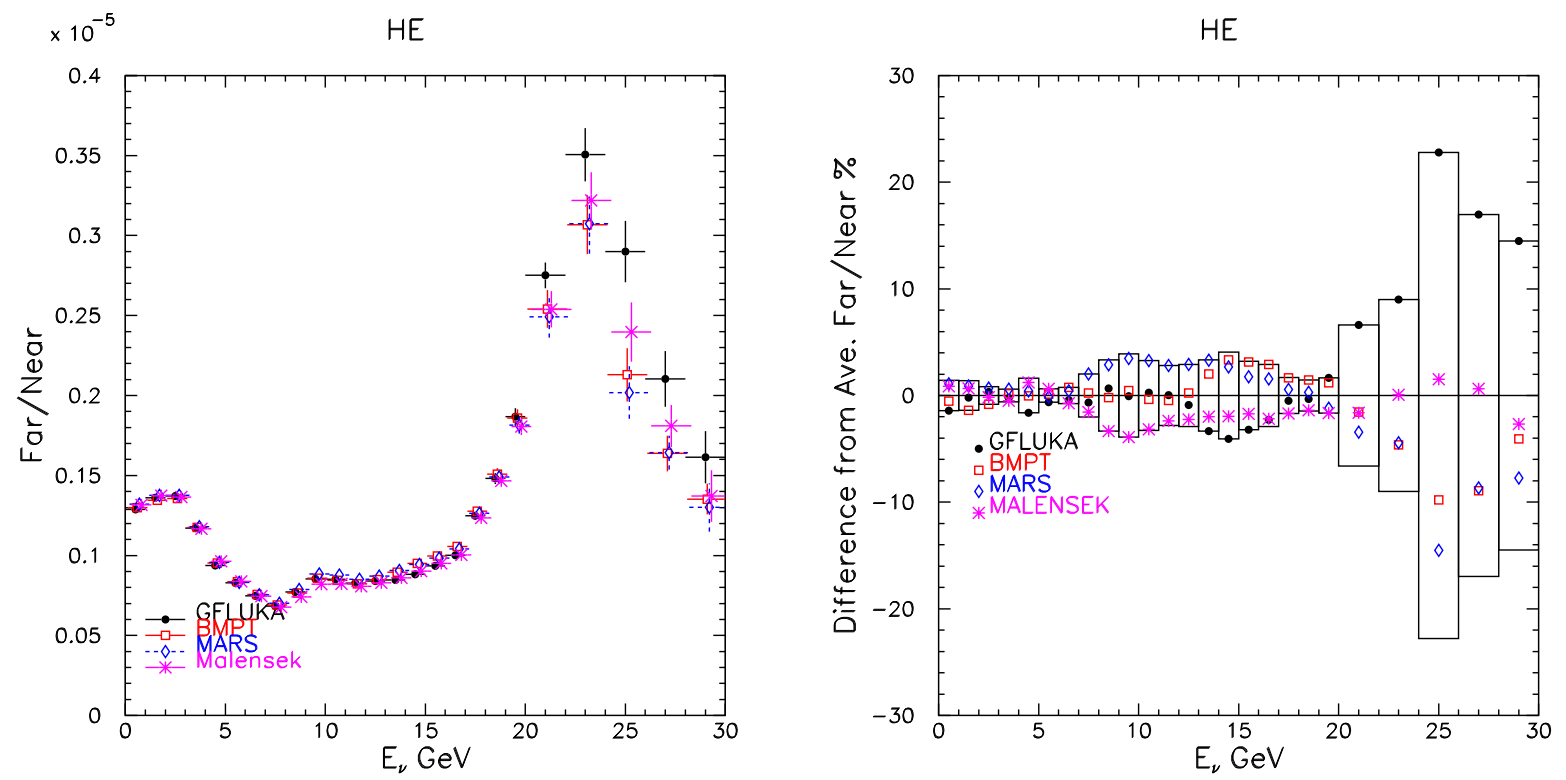

Figure 11: (left) The variation in far-over-near ratio expected due to different hadron production models in the HE beam. The lowest systematic spread from this source always occurs in the peak. (right) The $\mathrm{F} / \mathrm{N}$ of several models relative to that of Geant/Fluka.
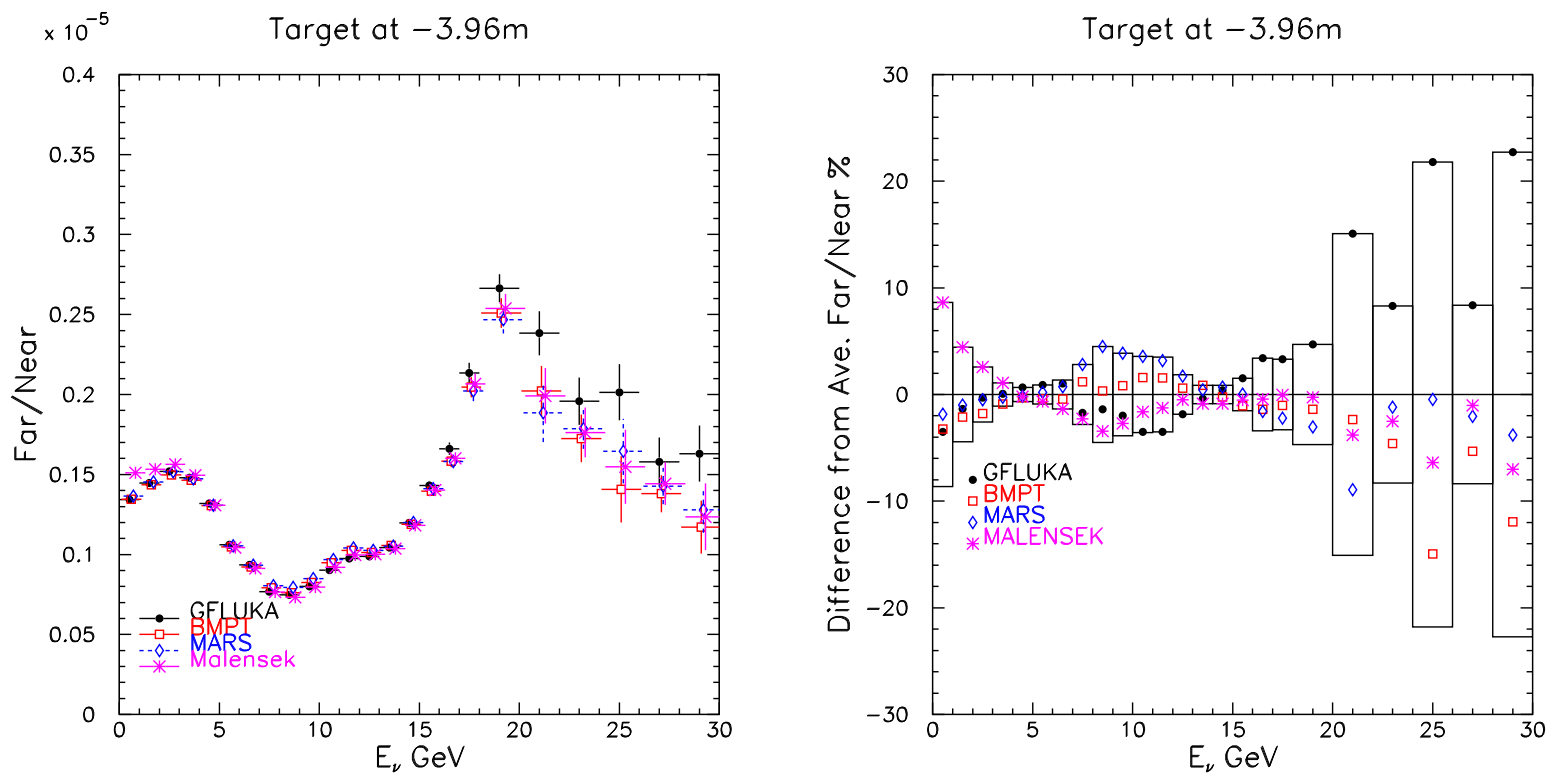

Figure 12: (left) The variation in far-over-near ratio expected due to different hadron production models in the semi-HE beam. The lowest systematic spread from this source always occurs in the peak. (right) The $\mathrm{F} / \mathrm{N}$ of several models relative to that of Geant/Fluka. 
Ratio of Ratios for Horn 1 moved by $3 \mathrm{~mm}$
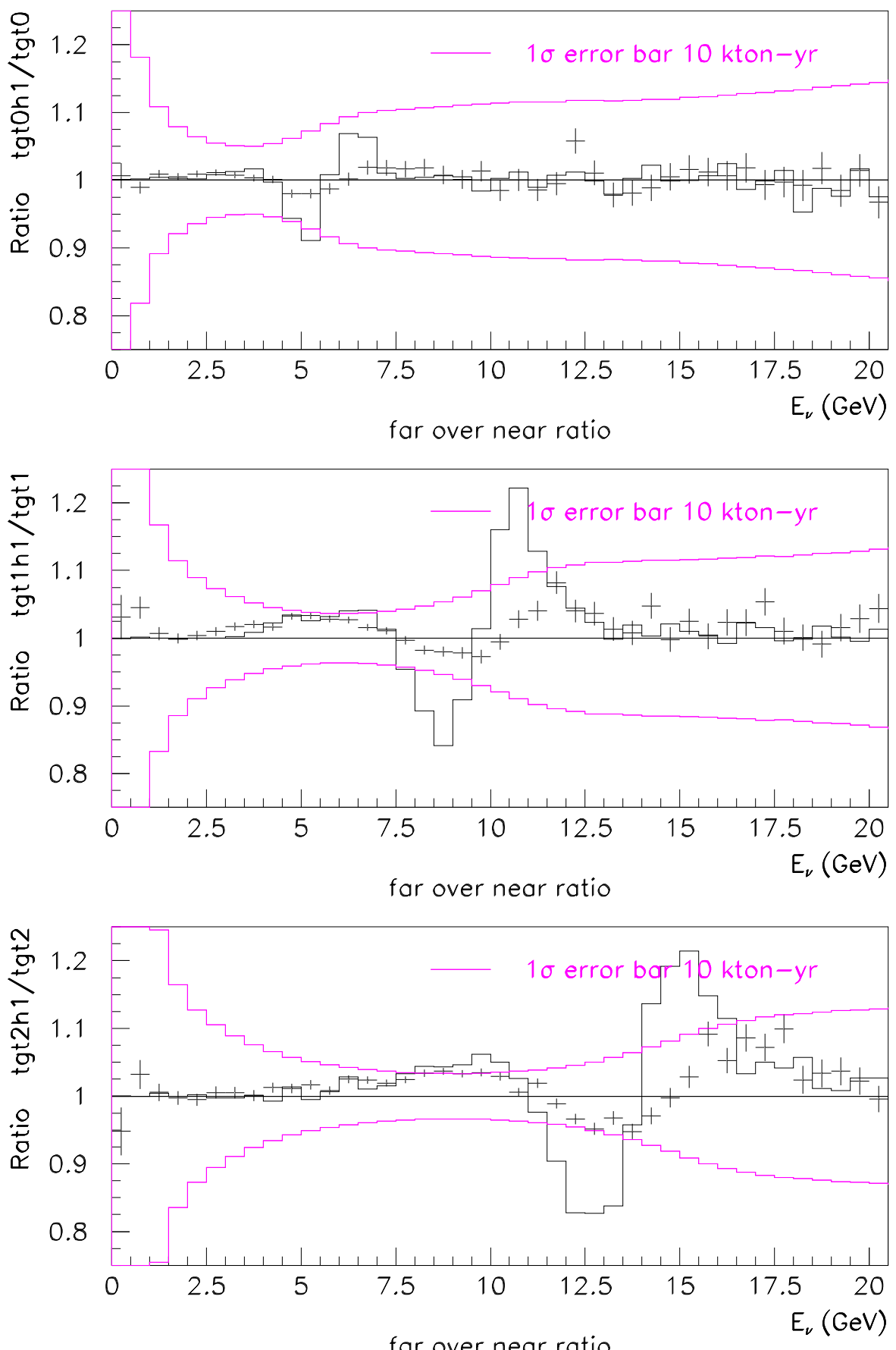

Figure 13: The variation in Far/Near due to misalignment of horn 1 by $3 \mathrm{~mm}$, as seen in the nominal LE beam (top), the semi-ME beam (middle), and the semi-HE beam (bottom). 

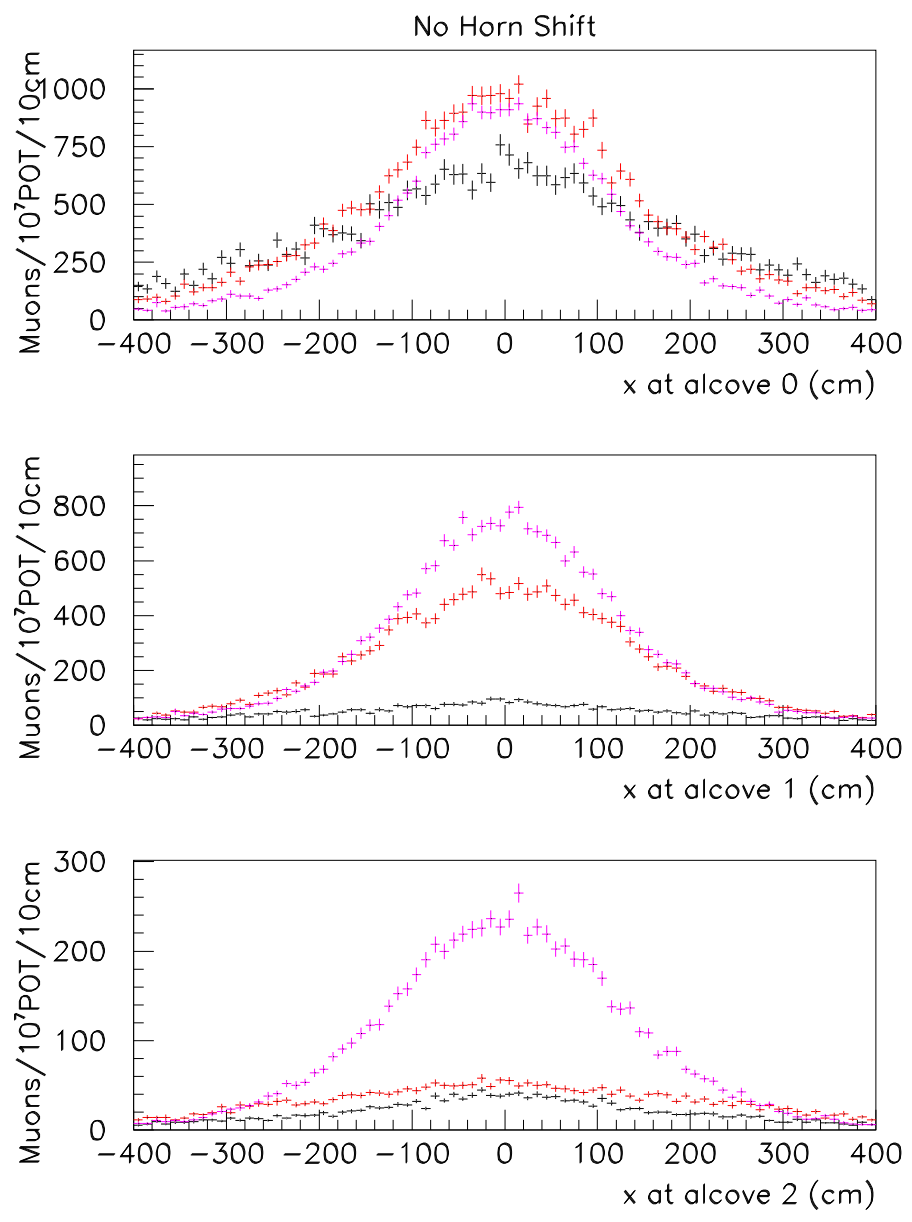

Figure 14: Muon distribution in the horizontal direction for the 3 alcoves when all beam elementa are perfectly aligned. The black points are for the nominal LE beam, the red points are the semi-ME beam, and the magenta points are the semi-HE beam.

\section{Monitoring and Commissioning of the Beam}

It is well-known that some of our more stringent alignment and design tolerances on the horns date from the period when the $\mathrm{HE}$ or ME beams were the baseline design. In the LE beam, the broad pion cloud that is being focused does loosen some of the tolerances, and some have noted that perhaps the original design specs are less relevant given our nominal LE beam choice. If, however, we wish to employ the semi-ME or semi-HE beams, those tolerances become quite relevant once again.

The monitoring system consisting of 3 muon alcoves and the downstream hadron monitor (DHM) is much less sensitive to beam misalignments, etc, in the LE beam precisely because of the broad distribution of muons coming from soft pion decays and also because of the 

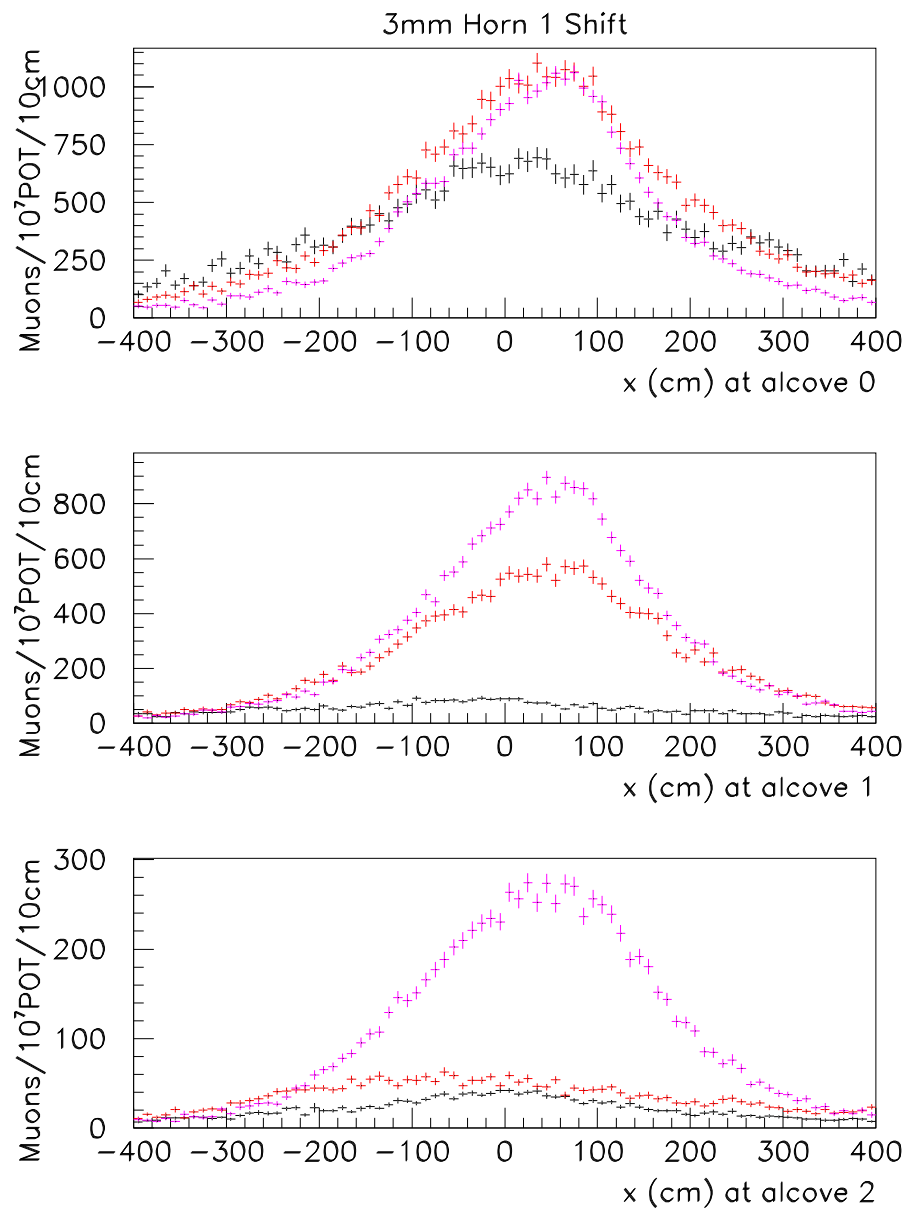

Figure 15: Muon distribution in the horizontal direction for the 3 alcoves when horn 1 is misaligned by $3 \mathrm{~mm}$. The black points are for the nominal LE beam, the red points are the semi-ME beam, and the magenta points are the semi-HE beam.

small acceptance of these detectors at the end of the decay pipe. However, it is well-known that the monitors are a much more powerful tool in the ME and HE beams, where the muons angles relative to the pion direction are much smaller.

On the following two pages are shown the same simulation for two conditions: in Figure 14 is the nominal beam, accurately aligned and properly pointing toward the Soudan mine, while in Figure 15 the 1st horn is shifted by $3 \mathrm{~mm}$. In both figures, the lateral distribution of muons in Alcoves 0, 1, and 2 are histogrammed. These muons were generated with the PBEAM Monte Carlo and swum to the alcoves. Only those muons above the stopping power of the $\mathrm{Al}$ in the hadron absorber or the dolomite rock get to the alcoves, and multiple scattering is applied.

For the purposes of this naive simulation, the absorber is treated as an aluminum block, 
which is quite simplified. The actual absorber has an aluminum core that extends $\pm 60 \mathrm{~cm}$ in the horizontal and vertical directions around the beam center. At larger transverse dimensions it is comprised of a non-uniform array of steel blocks which have a different muon momentum cutoff than the core region and is not uniform around its perimeter due to the varying number of blocks. Thus, the muon data outside of $\pm 60 \mathrm{~cm}$ in acceptance cannot be utilized to the same precision for pointing the beam. The area shadowed by the steel can only be used to track changes in the beam from spill-to-spill.

Both Figure 14 and 15 have 3 graphs. Each graph plots the lateral distribution of muons in the horizontal direction, one graph for each alcove. In each graph is shown the muon flux one would see in the nominal LE beam (black points), the semi-ME beam (red points), and the semi-HE beam (magenta points).

It is clear that as the beam energy is increased (target is pulled back in $z$ ), more (high energy) muons reach the latter two alcoves. It is also observed that the muons become more peaked. This peaking becomes visible even within the $\pm 60 \mathrm{~cm}$ window of the absorber Aluminum core, where one might know the material well enough to actually believe any peaking measurements made with the muon chambers are not due to absorber geometry. A powerful role of the monitoring system, then, could be to commission the beam in the semi-ME or semi-HE configuration by pointing the peak of Figure 14 toward Soudan. This pointing would require $a b$ initio channel-to-channel calibration prior to running the neutrino beam, as might be achieved using the in-situ alpha sources in the chambers. The precision of this calibration is still being determined.

Another way to use this information would be as a cross check of our beam simulations. As the neutrino beam energy is increased by moving the target, the flux gain in the downstream alcoves should increase owing to the greater number of higher energy muons from the decays of higher energy focused pions. The relative increase in the downstream alcoves should be predictable with our Monte Carlo, and would provide similar information as would other commissioning scans being discussed, such as gradually increasing the horn currents or pulsing just one horn during commissioning to focus different energy pions.

The semi-ME/HE beam also enhances the muon system's monitoring capabilities. If it would be possible to run mostly in the LE beam, but take occaisional runs in the semi-ME or semi-HE configurations, then these short runs could be used to check alignments, etc, of the horn optics. Figure 15, for example, shows the muon distribution one would see if horn 1 were displaced by $3 \mathrm{~mm}$ (admittedly an unlikely scenario, but demonstrative). Now the muon distribution is noticeably shifted, even within the $\pm 60 \mathrm{~cm}$ aluminum core of the absorber in which we can trust muon profiles the most. It would be clear during this short run that the horn optics were off and requiring some attention.

Discovering changes with these periodic semi-ME/HE monitoring runs actually makes less stringent use of the monitoring system. As the beam is switched from LE to semi$\mathrm{ME} / \mathrm{HE}$ position of the target, the shifted spectra of Figure 15 would appear, hence the monitoring system does not need to be able to be calibrated channel-to-channel; it merely needs to be track changes as the LE/semi-ME switch is made. This is further important because the material in the absorber outside of $\pm 60 \mathrm{~cm}$ may not be well known, so we 

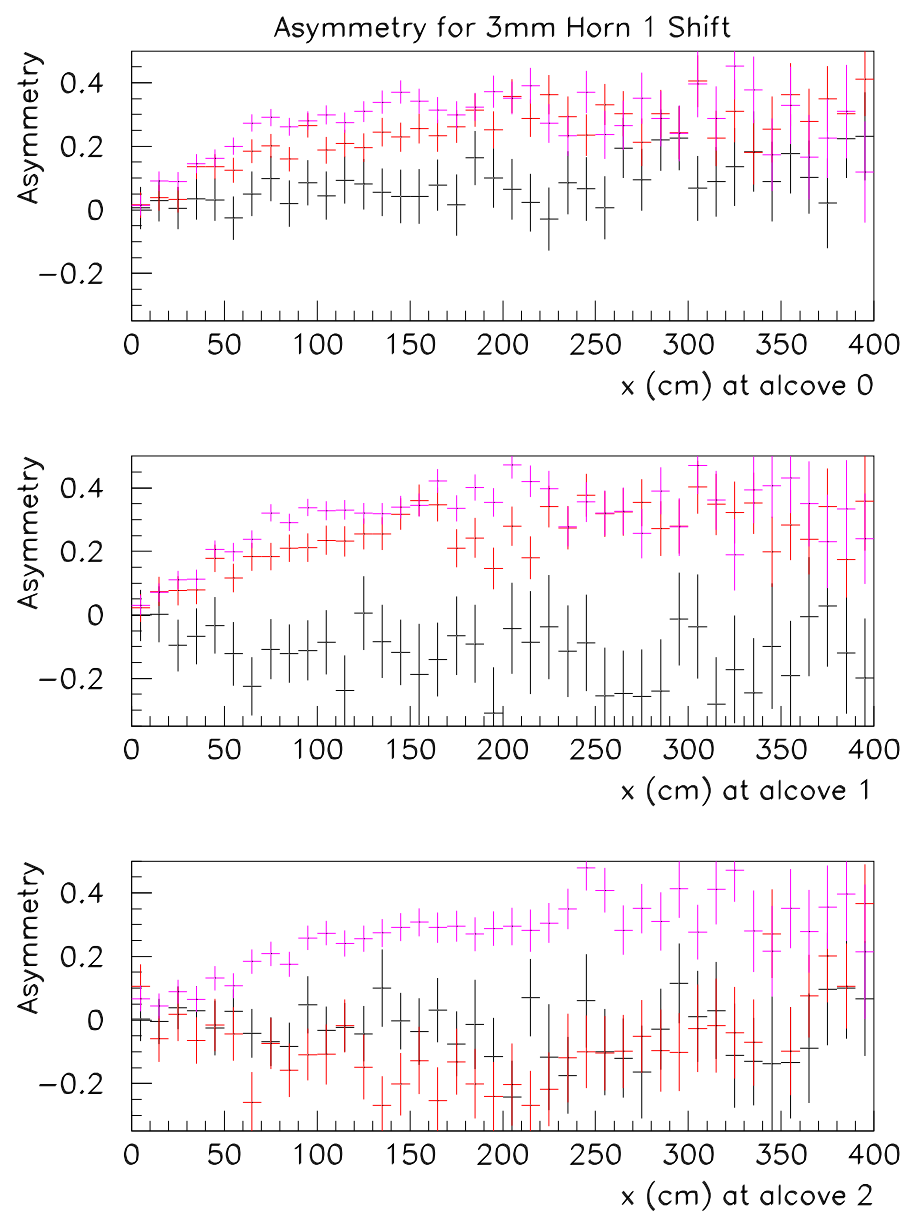

Figure 16: Muon rate asymmetry in the horizontal direction for the 3 alcoves when horn 1 is misaligned by $3 \mathrm{~mm}$. The black points are for the nominal LE beam, the red points are the semi-ME beam, and the magenta points are the semi-HE beam.

would use the monitoring system simply to measure the difference in rates between a $t=0$ semi-ME/HE run and the present semi-ME/HE run. Tracking changes with the monitoring system can be performed to within $\pm 1 \%$ [11]. Thus, the shifts in muon spectra all the way out to the $\pm 1 \mathrm{~m}$ area of the chanmbers are useful for monitoring our data. Some representative 'Asymmetries' derived from these plots are shown in Figure 16 


\section{Mechanics of Beam Energy Switch}

In the current design of the target pile, horn 1 is suspended from a 25 ton shielding module. The module provides four degrees of remote motion capability: the two directions transverse to the beam and the dip and horizontal angles. The range of motion is about $+/-8 \mathrm{~mm}$ at each end of the horn. The module is suspended from a carriage, which is a set of cross beams that extend the support point out to the concrete walls of the target pile. The concrete walls are thermally stable, so the horn will not move as the steel target pile heats up and expands.

Horn 2 is mounted in a similar fashion, except that the remote motion capability has been deleted as a cost saving measure.

The beam baffle and target are mounted together on a space frame, with the target remotely movable in the beam direction by about $1 \mathrm{~m}$. The space frame is suspended from a module nearly identical to the horn 1 module. The module provides the same 4 degrees of remote motion capability. The extra motion capability of the target in the beam direction is desired so that the target can be inserted into horn 1 in a delicate fashion after the modules are in place in the target pile and aligned. The baffle does not move in the beam direction, but could be moved out of the beam horizontally to allow a wide angle low intensity beam scan.

In the case of a failure of target or horn, the entire module in question is removed from the target pile to a work area with some remote manipulation capability (called the work cell). There, the failed component can be removed, and a replacement component can be installed on the module.

For movement from the LE to the Semi-ME beam, one would only need to use the remote motorized target motion along the beam line. This could be done in a few minutes, although one would presumably want to do a beam scan across the target after the movement to check the transverse coordinates in the new location.

For movement from the Semi-ME to the Semi-HE location, one would need to remove of order 30 shielding blocks, lift the module up, move the module about $2.5 \mathrm{~m}$ upstream, and set it back on the carriage. The carriage beams for the target module are long enough to accomodate the target module in either position without moving the carriage. After most of the shielding is replaced, one would do a survey to check the new module location. One could then replace the final shielding blocks, do a target scan with the beam, and proceed. The mechanics of the move might take 1 week. It might also be prudent to take another week to let the target pile radiation levels cool before beginning the movement, but this has not been studied.

To move horn 2 from the LE position to the ME or HE positions requires, in addition to moving the module, moving its carriage (or building a new carriage for it). It also requires installing special support rails for the associated shielding blocks. Since horn 2 does not have remote position capability, the survey/alignment of the carriage with the module in place is important. The move is complicated by the radiation environment. It could be considerably simplified by the pre-installation of the shielding rails and extra carriage, but this is not 
envisioned because of cost and schedule reasons. Additionally, strip-line to carry the $200 \mathrm{kA}$ horn current would have to be extended from the LE horn position to the new location. This change-over could take up to 2 months.

In summary, the semi-ME/HE beams are much easier to achieve than the nominal $\mathrm{ME} / \mathrm{HE}$ beams because horn 2 is not moved. Furthermore, if it is decided that having just the capability to scan the target 1 additional meter (to the semi-ME slot) is sufficient, this would be easier and cheaper to implement and motorize than to implement the flexibility for a remotely-scannable $4 \mathrm{~m}$ shift (semi-HE beam).

\section{Conclusions}

The simulations done for this note demonstrate a few points worth further study and consideration. They include:

- One does not need to telescope horn 2 to achieve a ME or HE beam. Semi-ME or Semi-HE beams can increase the neutrino energy and have the advantage of being easier to switch to.

- Using just the LE target for all 3 beams also affords the possibility of more rapid transitions between LE, ME, and HE beams.

- The semi-ME/HE beams could be used to obtain a low-systematic measurement of the 6-20 GeV region of the neutrino energy spectrum which complements the lowsystematics region from $0-6 \mathrm{GeV}$ in the LE beam.

- Rapid switching between LE and semi-ME/HE beams has utility for beam commissioning as far as pointing the neutrino beam.

- Although requiring further study, it appears monitoring the LE beam could be strengthened by occaisionally running in the semi-ME/HE configuration since to see if the beam/horn optics have changed.

- In a separate note, it was proposed to build an extension to the decay pipe to be added which increases the LE beam event rate by $10 \%$ [2]. While the variable energy beam is unrelated to this other proposal, its potential utility might also allow the decay pipe extension to be further considered since the ME/HE beams would not be precluded.

Thus it is hoped that these simulations will stimulate further investigation as to how to best commission the beam, how to best monitor the beam during LE running, and how to run for $10 \mathrm{kt}-\mathrm{yr}$ at $4 \mathrm{E} 20$ protons on target per year to best achieve MINOS' physics goals. Some example points for discussion would be (1) given that $1 \mathrm{~m}$ target shifts are easily accomodated in the present design and $>1 \mathrm{~m}$ requires greater modification, would going to only the semi-ME beam be sufficient, or is the semi-HE position of greater additional value?

(2) how would the collaboration weight its physics data set in the LE/semi-ME/semi-HE 
modes? (3) what is the frequency of taking periodic monitoring runs? (4) what is the commissioning plan? (5) how could the target position be remotely verified after it has been relocated? It is beyond the scope of this note to address all such points, and we hope that this note will stimulate future discussions on the potential utility of variable energy beams.

\section{References}

[1] Hylen, J., et al., "Conceptual design for the technical components of the neutrino beam for the main injector (NuMI)," Fermilab-TM-2018 (1997).

[2] M. Kostin et al, "Effect of Decay Pipe Window, Vacuum on Neutrino Fluxes", NuMIB-777, September, 2001.

[3] Gnumi version 14.0 was checked into the CVS repository July 28, 2001. Mark Messier presented results at the July Beam Monte Carlo workshop discussing the geometry changes.

[4] S.Kopp et al, "Hadron Production Models, Revisted" NuMI-B-768, July, 2001.

[5] N.V. Mokhov, "The MARS Monte Carlo", Fermilab FN-628 (1995); O.E. Krivosheev et al., Proc. of the Third and Fourth Workshops on Simulating Accelerator Radiation Environments (SARE3 and SARE4), Fermilab-Conf-98/043(1998) and Fermilab-Conf98/379(1998).

[6] GEANT Detector Description and Simulation Tool, CERN Program Library, W5013 (1994).

[7] M. Bonesini, A. Marchionni, F. Pietropaolo, and T. Tabarelli de Fatis, "On Particle Production for High Energy Neutrino Beams," Eur. Phys. J. C20, 13-27 (2001).

[8] A.J. Malensek, "Empirical Formula for Thick Target Particle Production," Fermilab FN-341, Oct. 1981.

[9] G. Collazuol, A. Ferrari, A. Guglielmi, P.R. Sala, Nucl. Instr. Meth. 449, 609 (2000). A. Ferrari and P.R. Sala, ATLAS Internal Note ATL-PHYS-97-113, Proc. of the Workshop on Nuclear Reaction Data and Nuclear Reactors Physics, Design and Safety, ICTP, Trieste, Italy 1996. Publ. by World Scientific, A.Gandini, G.Reffo, eds.

[10] P. Sala, talk given at the $2^{n} d$ International Workshop on Neutrino Beams and Instrumentation, 6-9 September, 2000, Batavia, IL.

[11] Debbie Harris, NuMI-B-756, "Specifications for the Muon Monitoring System", May, 2001. 